\title{
ERRATUM
}

\section{VOLUME 17}

Page 489. The sentence on line 3-4 of the article Early history of the Generalized Continuum Hypothesis: 1878-1938 by GREGORY H. Moore contains a word which became corrupted after proofreading was finished. It should read as follows:

However, the origins and early history of the Generalized Continuum Hypothesis have not. 